\title{
Association of Urinary Albumin:Creatinine Ratio with Outcome of Children with Sepsis
}

\author{
Anil Sachdev ${ }^{1}$, Karan Raheja ${ }^{2}$, Neeraj Gupta ${ }^{3}$, Parul Chugh $^{4}$
}

\begin{abstract}
Objective: The aim of the study was to investigate the association of urinary albumin:creatinine ratio (ACR) with regard to the outcome of sepsis patients and to study the trends of ACR with severity of disease, organ dysfunction, microcirculation status, the use of inotrope, and mechanical ventilation use, and length of pediatric intensive care unit (PICU) stay.

Materials and methods: In the prospective observational study, the patients with varying categories of sepsis admitted in the PICU with stay $>24$ hours were enrolled consecutively. Urine samples were collected at the time of admission (ACR1), 12 hours (ACR2), and 24 hours (ACR3). Results: One hundred and thirty-eight patients including 56 cases of sepsis, 31 of severe sepsis, 22 of septic shock, and 29 of multiorgan dysfunction syndrome (MODS) cases were analyzed. There were 29 (21\%) deaths. ACR (median, IQR) was significantly higher in nonsurvivors [ACR1 198.9 (111.2-329.4) vs 124.5 (59.37-294.5), p 0.03], [ACR2 213.8 (112.5-350) vs 117.8 (62.6-211.9) p 0.008], [ACR3 231.8 (99.9-441.2 vs 114.4 (44.1-240.3), $p$ 0.005]. The ACR is increased progressively with the increasing severity of sepsis $(p<0.001)$. The performance of ACR operative characteristics was compared with that of PRISM and PELOD scores. In deceased, ACR was significantly correlated with blood pH, lactate, and base deficit. A cutoff value of ACR $102.7 \mathrm{mg} / \mathrm{g}$ had sensitivity $86.2 \%$, specificity $40.4 \%$, positive predictive value $27.8 \%$, and negative predictive value $91.7 \%$. The use of inotropes, mechanical ventilation ( $>48$ hours), and mortality was significantly higher in patients with ACR $>102 \mathrm{mg} / \mathrm{g}$. The probability of death varied from 17.6 to $19 \%$ in the first 24 hours of admission. ACR was significantly cheaper as compared to PRISM score and PELOD score estimations.

Conclusion: Urinary ACR, a cost-effective tool, correlates with the severity of sepsis and associated morbidity and mortality in children.

Keywords: Microalbuminuria, Mortality, Multiorgan system failure, Outcome prediction, Pediatric intensive care, Sepsis.

Indian Journal of Critical Care Medicine (2020): 10.5005/jp-journals-10071-23463
\end{abstract}

\section{INTRODUCTION}

Patients requiring intensive care frequently have some degree of systemic inflammatory response syndrome (SIRS), which, when severe, places them at risk of multiple organ failure. ${ }^{1,2}$ An early feature of the acute inflammatory process is capillary endothelial cell activation accompanied by a rapid increase in capillary permeability to plasma proteins such as albumin. The transcapillary escape rate of radio-labeled albumin from the circulation increases dramatically within 3 hours of cardiac surgery in patients with infection and malignancies. ${ }^{3,4}$ Studies in normal subjects and in patients with diabetes mellitus or hypertension have also demonstrated an association between urine albumin and albumin transcapillary escape rate and evidence of systemic endothelial dysfunction., The presence of microalbuminuria (MAU) has been associated with postoperative pulmonary complications, injury severity, and inflammatory insult following trauma, ischemia reperfusion injury, pancreatitis, meningitis, anaphylactic shock, and inflammatory bowel disease. ${ }^{2,7-17}$ All these studies are consistent with the concept that MAU is associated with systemic microvascular dysfunction. Significant MAU has been demonstrated in adults and children with sepsis as compared to non-septic patients. ${ }^{17-20}$ We did not find any study in children and adults where MAU was estimated in the different categories of sepsis, severe sepsis, septic shock, and multiorgan dysfunction syndrome (MODS). It was hypothesized that the higher level of urinary albumin:creatinine ratio (ACR) was corresponded with increasing the severity of disease and organ dysfunction in children suffering with sepsis, severe sepsis, septic shock, and MODS and will also predict mortality and morbidity.

\begin{abstract}
1,3Pediatric Emergency, Critical Care and Pulmonology, Department of Pediatrics, Sir Ganga Ram Hospital, New Delhi, India

${ }^{2}$ Pediatric Emergency and Critical Care, Department of Pediatrics, Sir Ganga Ram Hospital, New Delhi, India

${ }^{4}$ Department of Research, Sir Ganga Ram Hospital, New Delhi, India

Corresponding Author: Anil Sachdev, Pediatric Emergency, Critical Care and Pulmonology, Department of Pediatrics, Sir Ganga Ram Hospital, New Delhi, India, Phone: +91 9810098360, e-mail: anilcriticare@ gmail.com
\end{abstract}

How to cite this article: Sachdev A, Raheja K, Gupta N, Chugh P. Association of Urinary Albumin:Creatinine Ratio with Outcome of Children with Sepsis. Indian J Crit Care Med 2020;24(6):465-472.

Source of support: Nil

Conflict of interest: None

The primary objective of study was to investigate the predictive value of ACR with regard to the outcome of the patients with sepsis. The secondary objectives were to study the trends of urinary ACR with the severity of disease, organ dysfunction, microcirculation status, use of inotrope, mechanical ventilation, and the length of PICU stay in the patients suffering with varying severity of sepsis and MODS.

\section{Materials and Methods}

Consecutive patients with suspected sepsis admitted to 12-bed pediatric intensive care unit (PICU) in a tertiary care teaching

() The Author(s). 2020 Open Access This article is distributed under the terms of the Creative Commons Attribution 4.0 International License (https://creativecommons. org/licenses/by-nc/4.0/), which permits unrestricted use, distribution, and non-commercial reproduction in any medium, provided you give appropriate credit to the original author(s) and the source, provide a link to the Creative Commons license, and indicate if changes were made. The Creative Commons Public Domain Dedication waiver (http://creativecommons.org/publicdomain/zero/1.0/) applies to the data made available in this article, unless otherwise stated. 
hospital were enrolled over 14 months from August 2017 to October 2018. The institutional research and the ethics committee approved the study protocol. A written consent was obtained from the parents after explaining the purpose of sending urine samples for urinary ACR. All the pediatric patients with sepsis and with a stay in PICU for more than 24 hours were included. It was determined a priori that patients would be excluded if they had confounding factors such as anuria, macroscopic hematuria, diabetes mellitus, preexisting chronic kidney disease, urinary tract infection (UTI), proteinuria, hypertension, pigmented urine, and inability to collect urine. There was no additional financial burden on the parents for ACR estimation.

\section{Patient Demography}

At the time of admission, the following data were collected for each patient: age, gender, date and time of admission, patient's clinical classification (medical or surgical), provisional diagnosis, comorbid conditions such as diabetes mellitus, hypertension, and chronic kidney disease. Clinical and relevant laboratory data were also collected. The patients were divided into different categoriessepsis, severe sepsis, septic shock, and MODS. ${ }^{21}$ For disease severity scoring, PRISM scores at 12 and 24 hours were calculated, and for organ dysfunction, PELOD scores were calculated at the time of admission, 12 hours, and 24 hours of admission. ${ }^{22,23}$ Each patient was followed up through their PICU stay for a maximum of 28 days.

\section{Sample Collection and Laboratory Measurement}

Spot urine samples were collected by the PICU nurses at the time of admission, 12 hours and 24 hours for quantification of ACR, which were referred to as ACR1, ACR2, and ACR3, respectively. The urine samples were delivered to biochemistry laboratory and stored at $-20^{\circ} \mathrm{C}$ till analysis. The urinary microalbumin level was measured using the immune-turbidimetric method (Dimension RxL Max, Dade Behring Inc., USA). Urine creatinine was measured using a modified kinetic Jaffe reaction (Dimension RxL Max, Dade Behring Inc., USA). The methods covered an analytical range of 1.3 to $100 \mathrm{mg} / \mathrm{L}$ for microalbumin. The results obtained beyond the analytical range were diluted appropriately and were rerun. The MAU, expressed as ACR in $\mathrm{mg} / \mathrm{g}$ of creatinine, is defined as ACR of $<300 \mathrm{mg} / \mathrm{g}$. The ratio has a conventional cutoff value of $30 \mathrm{mg} / \mathrm{g}$ in the healthy reference population. The reference range for mortality prediction in critically ill children is yet to be determined. ${ }^{24}$ The vasopressor-inotrope score was calculated with the formula used previously by Gaies et al. ${ }^{25}$

\section{Statistical Analysis}

The statistical analysis was performed by the SPSS program for Windows, version 17.0 (SPSS, Chicago, Illinois). The data were checked for normality before the statistical analysis. The descriptive statistics were used to describe the data. The ACR measured at different time points was correlated with various parameters and scores using Spearman's correlation coefficient. The receiver operating characteristic (ROC) curves and area under the curve (AUC) were determined and their operative characteristics were calculated. The univariate analysis, multivariate analysis, and log transformation were used to identify the potential risk factors associated with the outcome. For all the statistical tests, $p$ value less than 0.05 was considered significant.

\section{Results}

Two hundred and eighty-five patients with suspected sepsis were admitted at the PICU during the study period. All the 138 patients fulfilling the inclusion criteria were enrolled (Flowchart 1). The median age and weight of study cohort were 14.5 (IQR 4-50.5) months and 8.2 (IQR 4.8-18) kg, respectively. There were 66 (47.8\%) cases $\leq 12$ months of age. The median values of PRISM score at 12 and 24 hours were 7 (IQR 3-10) and 6 (IQR 1-8) and PELOD score at the time of admission, 12 hours, and 24 hours of admission were 12 (IQR 2-24), 12 (IQR 1-22) and 12 (IQR 1-21), respectively. One hundred and five (76.6\%) patients required invasive mechanical ventilation at the time of admission and 93 patients were ventilated for more than 48 hours. The median length of stay in PICU was 6 (IQR 3.7-12) days. The respiratory system was the commonest primary organ involved in $50(36.8 \%)$ cases. Primary sepsis was present in 24 (17.4\%) while gastrointestinal and liver infections were present in 18 (13\%) cases. Tropical diseases with sepsis were diagnosed in 19 cases. Rest of the cases had primary neurological infections in 11 children, and skin, soft tissue, and musculoskeletal infections in 10 cases. There were only six cases with myocarditis admitted in the PICU since there is a separate pediatric cardiac ICU in the hospital. Forty seven (34\%) children had blood culture positive at the time of admission. There were 29 (21\%) deaths in the study cohort (Table 1).

\section{Albumin:Creatinine Ratio and Disease Severity and Organ Dysfunction}

Twenty-nine urine samples revealed normal range of albumin excretion. Twenty-three samples belonged to sepsis category,

Flowchart 1: Study flowchart. UTI, urinary tract infection; PICU, pediatric intensive care unit

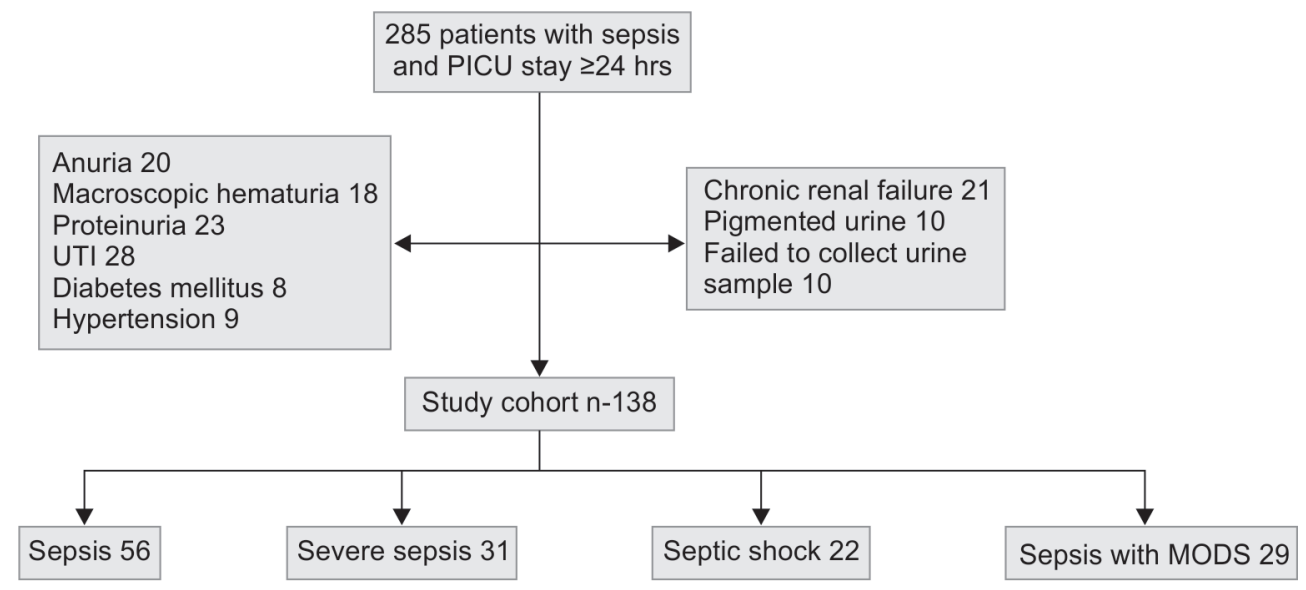


Table 1: Comparison of survivor and nonsurvivors

\begin{tabular}{llll}
\hline Parameter & $\begin{array}{l}\text { Survivors } \\
(n=29)\end{array}$ & $\begin{array}{l}\text { Nonsurvivors } \\
(n=109)\end{array}$ & $p$ value \\
\hline${\text { Age }(\text { month })^{*}}$ & $16(3.5-60)^{*}$ & $11(4.5-28)$ & 0.5 \\
Male $^{* *}$ & $85(78)^{* *}$ & $25(86)$ & 0.4 \\
Weight (kg) & $10(4.6-18)$ & $7(5-12)$ & 0.4 \\
PRISM 12 & $6.5(2-9)$ & $9(4-13.5)$ & 0.02 \\
PRISM 24 & $4(0.5-8)$ & $8(5.5-14)$ & $<0.01$ \\
PELODS 0 & $12(10-22)$ & $22(12-31)$ & 0.01 \\
PELODS 12 & $12(2-21)$ & $21(10.5-27)$ & 0.007 \\
PELODS 24 & $11(0-21)$ & $21(12-28)$ & $<0.01$ \\
Arterial pH 0*** & $7.30 \pm 0.1$ & $7.23 \pm 0.13$ & 0.005 \\
Blood lactate 0 & $1.95 \pm 1.11$ & $2.55 \pm 1.04$ & 0.02 \\
Blood glucose 0 & $112.5 \pm 36.4$ & $134.2 \pm 70.2$ & 0.2 \\
ACR1 & $124.5(59.37-294.5)$ & $198.9(111.2-329.4)$ & 0.031 \\
ACR2 & $117.8(62.6-211.9)$ & $213.8(112.5-350)$ & 0.008 \\
ACR3 & $114.4(44.1-240.3)$ & $231.8(99.9-441.2)$ & 0.005 \\
MV & $76(69.7)$ & $29(100)$ & $<0.01$ \\
VIS & $0(0-10)$ & $76(5-89.5)$ & $<0.01$ \\
LOS PICU (d) & $6(3-12)$ & $6(4-12.5)$ & 0.5 \\
Sepsis & $54(49.5)$ & $2(6.8)$ & 0.0001 \\
Severe sepsis & $27(24.7)$ & $4(13.7)$ & 0.20 \\
Septic shock & $17(15.5)$ & $5(17.2)$ & 0.82 \\
MODS & $11(10)$ & $18(62)$ & $<0.001$ \\
\hline
\end{tabular}

*Median with interquartile range, ${ }^{* *}$ Frequency with percentage, ${ }^{* * *}$ Mean $\pm \mathrm{SD}$

PRISM, pediatric risk of mortality; PELODS, pediatric logistic organ dysfunction score; arterial blood $\mathrm{pH}$, and lactate level in $\mathrm{mmol} / \mathrm{L}$ at the time of admission, blood glucose at the time of admission in $\mathrm{mg} / \mathrm{dL}, \mathrm{ACR} 1$, ACR2, ACR3 albumin:creatinine ratio ( $\mathrm{mg} / \mathrm{g}$ of creatinine) at the time of admission, 12 hours and 24 hours, respectively; MV, mechanical ventilation; VIS, vasopressor-inotrope score; LOS, length of stay in days; PICU, pediatric intensive care unit; MODS, multiorgan dysfunction syndrome

four were of severe sepsis and one each belonged to septic shock and MODS patients. The age and gender distribution were not significantly different among different categories of sepsis. There were significantly higher ACR1, ACR2 and ACR3 levels in the patients with severe sepsis, septic shock, and in patients with MODS as compared to sepsis cases (Table 2). The AUCs of ROC curves of PRISM 12, PRISM 24, and PELOD scores at the time of admission, 12 hours, and at 24 hours of admission to predict mortality were comparable with those of ACR1, ACR2, and ACR3 (Table 3). The ACR at three time points was correlated significantly with respective PELOD score at the time of admission, 12 hours, and 24 hours of admission in alive and expired patients, whereas ACR2 was correlated with PRISM 12 in both survivors and expired while ACR3 was correlated with PRISM 24 only in survivors and not in deceased (Table 4).

\section{Microalbuminuria and Microcirculation}

Arterial blood $\mathrm{pH}$, base deficit, and lactate levels are the indicators of tissue microcirculation. ACR1, ACR2, and ACR3 had consistent and strong correlation with arterial blood $\mathrm{pH}$, base deficit, and lactate levels obtained at the time of admission, 12 hours, and 24 hours of admission, respectively, in nonsurvivors (Table 4), while in survivors, these correlations were inconsistent and insignificant.

\section{Morbidity and Mortality Analysis}

Fifty cases had worsening trends of ACR levels over 24 hours and 18 (36\%) cases died, whereas there were only 12 (13.6\%) deaths in 88 cases with improving the trends of ACR ( $p$ value 0.002 ). ACR1, ACR2, and ACR3 were consistently higher in nonsurvivors as compared to survivors (Table 1). There was a significant fall in ACR1 and ACR2 ( $p$ value 0.008 ) and ACR1 and ACR3 ( $p$ value 0.016 ) in survivors, while there were consistently increasing trends of ACR values in nonsurvivors without statistical significance (Fig. 1). The operative characteristic analysis of ACR1 values revealed the level of 102.7 $\mathrm{mg} / \mathrm{m}$ with sensitivity $86.2 \%$, specificity $40.4 \%$, positive predictive value $27.8 \%$, and negative predictive value $91.7 \%$. Further analysis of urinary ACR values (ACR1, ACR2, ACR3) with cutoff value of 102 $\mathrm{mg} / \mathrm{g}$ was done (Table 5). The use of inotropes and mechanical ventilation at the time of admission, number of inotropes, duration of the ventilation for $\geq 48$ hours, and mortality were significantly higher in the patients with $A C R>102 \mathrm{mg} / \mathrm{g}$.

Figure 2 depicts the predicted probability of death with increasing levels of ACR after the admission in PICU. The probability of death varied from 17.6 to $19 \%$ with ACR level of $102 \mathrm{mg} / \mathrm{g}$ in the first 24 hours of admission.

\section{Cost Analysis}

There is a need of six laboratory parameters to calculate PRISM score and five variables are required to calculate PELOD score. The costs of estimating PRISM score, PELOD score, and ACR in our hospital were Rs 2545 (US\$ 37.5), 2620 (US \$ 38.5), and 375 (US \$ 5.5), respectively.

\section{Discussion}

The present study was done on consecutive children admitted to the PICU with varying severity of sepsis including sepsis, severe sepsis, septic shock, and multiorgan dysfunction. The urinary ACR was estimated at three time points-at the time of admission, 12 hours, and 24 hours of admission. The ACR showed rising trends with increasing severity of sepsis. Serial levels of ACR were consistently higher in nonsurvivors and there was a significant drop in ACR in survivors in the first 24 hours of admission. The ACR levels were correlated with PRISM 12 and 24 score and PELOD score. This was the first study to demonstrate significant correlation between serial levels of ACR and arterial blood $\mathrm{pH}$, serum lactate, and base deficit particularly in nonsurvivors. We also found a threshold level of ACR $>102 \mathrm{mg} / \mathrm{g}$ to correlate with the prolonged duration of the mechanical ventilation and need for more number of inotropes and higher mortality.

A very early feature of inflammation is rapid increase in capillary permeability to plasma protein (within a few minutes) following injury, and it usually returns to normal within 48 hours unless complications occur. ${ }^{3}$ Since renal tubules for albumin absorption are near saturation, $1 \%$ increase in vascular permeability is amplified by kidney and converted into doubling the urinary albumin excretion. ${ }^{26}$ It has been suggested that the timing of evaluation is an important determinant of predictive value of MAU in critically ill patients. ${ }^{27,28}$ In the present study, the first urine sample for evaluation was collected at the time of admission in the PICU either directly or after catheterization within mean time of 55 minutes ( \pm 26 minute).

The MAU can be assessed by calculating ACR in a spot urine sample as done in the present study. This ratio takes into account the differences in urine volume and most closely approximate with gold standard, 24-hour urine albumin excretion. ${ }^{29}$ In large 
Table 2: Patient characteristics in different categories of sepsis

\begin{tabular}{|c|c|c|c|c|c|}
\hline Variable & Sepsis $(n=56)$ & Severe sepsis $(n=31)$ & Septic shock $(n=22)$ & $\operatorname{MODS}(n=29)$ & $p$ value \\
\hline PRISM 12a & $4(1.2-7.7)$ & $8(6-11.2)$ & $9.5(7-13.2)$ & $8(5-13.5)$ & $<0.001$ \\
\hline PRISM 24 & $2(0-5.5)$ & $6(5-10)$ & $6.5(1.5-9.2)$ & $8(5.5-12)$ & $<0.001$ \\
\hline PELODS 0 & $10(1-12)$ & $21(12-31)$ & $21.5(12-31)$ & $22(12-32)$ & $<0.01$ \\
\hline PELODS 12 & $2(1-12.7)$ & $21(12-24)$ & $13(9.2-22$ & $21(10-27)$ & $<0.01$ \\
\hline PELODS 24 & $1(0-11.7)$ & $21(11-22)$ & $12.5(9.2-22.2)$ & $21(11.5-22.5)$ & $<0.001$ \\
\hline Blood $\mathrm{pH} 0^{* \mathrm{~b}}$ & $7.34 \pm 0.78$ & $7.24 \pm 0.12$ & $7.27 \pm 0.13$ & $7.23 \pm 0.11$ & $<0.001$ \\
\hline $\mathrm{pH} 12$ & $7.35 \pm 0.84$ & $7.30 \pm 0.11$ & $7.30 \pm 0.08$ & $7.24 \pm 0.11$ & $<0.001$ \\
\hline pH 24 & $7.37 \pm 0.63$ & $7.33 \pm 0.08$ & $7.35 \pm 0.1$ & $7.29 \pm 0.1$ & 0.001 \\
\hline Lactate $0^{*}$ & $1.78 \pm 0.71$ & $2.0 \pm 0.91$ & $2.4 \pm 1.74$ & $2.5 \pm 1.26$ & 0.015 \\
\hline Lactate 12 & $1.57 \pm 0.67$ & $1.95 \pm 1.27$ & $2.03 \pm 0.79$ & $2.39 \pm 0.84$ & 0.001 \\
\hline Lactate 24 & $1.52 \pm 0.58$ & $1.59 \pm 0.71$ & $1.90 \pm 0.73$ & $1.96 \pm 0.84$ & 0.019 \\
\hline VIS & $0(0-0)$ & $0(0-0)$ & $25(10-30$ & $70(50-89.5)$ & $<0.001$ \\
\hline MV use ${ }^{c}$ & $28(50)$ & $28(90.3)$ & $21(95.50)$ & $28(96.6)$ & $<0.001$ \\
\hline ACR1 & 79.5 (33.7-127.4) & $269.8(139.2-528.5)$ & $154.5(103.8-333.5)$ & $161(116-329.4)$ & $<0.001$ \\
\hline ACR2 & $73.2(30.1-130.8)$ & $187.9(123.8-344.2)$ & $119.4(94.4-352.6)$ & $196.3(112.7-338.2)$ & $<0.001$ \\
\hline ACR3 & $60.4(22.9-1118.2)$ & $198.7(119.8-321.8)$ & $119.4(83.7-321.6)$ & $196.3(118.5-400.8)$ & $<0.001$ \\
\hline $\mathrm{PICU}^{* *}$ & $6(3-12)$ & $6(4-10)$ & $6.5(4.7-12.2)$ & $8(4-16)$ & 0.18 \\
\hline Mortality & $2(3.5)$ & $4(12.9)$ & $5(22.7)$ & $18(62)$ & $<0.001$ \\
\hline
\end{tabular}

*Arterial blood sample at the time of admission, 12 and 24 hours in mmol/L; PICU, pediatric intensive care unit; ** Duration of stay in PICU is in days. PRISM, pediatric risk of mortality score; PELOD, pediatric logistic organ dysfunction score; ACR1, ACR2, ACR3 albumin:creatinine ratio (mg/g of creatinine) at the time of admission, 12 and 24 hours, respectively; VIS, vasopressor-inotrope score; MV, mechanical ventilation; MODS, multiorgan dysfunction syndrome; LOS, length of stay in days; PICU, pediatric intensive care unit. The difference of ACR values between severe sepsis and septic shock was not statistically significant

${ }^{\mathrm{a}}$ Median with interquartile range

${ }^{\mathrm{b}}$ Mean $\pm \mathrm{SD}$

${ }^{\mathrm{C}}$ Frequency with percentage

Table 3: Receiver operative characteristics with area under the curve of albumin:creatinine ratio, disease severity and organ dysfunction scores to predict mortality

\begin{tabular}{llcl}
\hline & $\begin{array}{l}\text { Area under } \\
\text { the curve }\end{array}$ & p value & $\begin{array}{l}\text { 95\% confidence } \\
\text { interval }\end{array}$ \\
\hline ACR1 & 0.612 & 0.03 & $0.494-0.709$ \\
ACR2 & 0.661 & 0.008 & $0.554-0.767$ \\
ACR3 & 0.669 & 0.005 & $0.560-0.778$ \\
PRISM score 12 & 0.639 & 0.021 & $0.522-0.757$ \\
PRISM score 24 & 0.737 & $<0.001$ & $0.633-0.843$ \\
PELOD score 0 & 0.665 & 0.007 & $0.552-0.778$ \\
PELOD score 12 & 0.663 & 0.007 & $0.545-0.781$ \\
PELOD score 24 & 0.709 & 0.001 & $0.599-0.820$ \\
\hline ACR1, ACR2,
\end{tabular}

ACR1, ACR2, ACR3 albumin:creatinine ratio ( $\mathrm{mg} / \mathrm{g}$ of creatinine) at the time of admission, 12 hours, and 24 hours of admission, respectively; PRISM, pediatric risk of mortality at 12 hours and 24 hours of admission; PELODS, pediatric logistic organ dysfunction score at the time of admission, 12 hours, and 24 hours of admission

population-based studies, MAU in children and adolescents is defined as an urinary albumin excretion rate of $30-300 \mathrm{mg} / 24$ hours in a 24-hours urine collection. ${ }^{30}$ However, it is not possible to assess urinary albumin excretion in 24 hour sample and particularly in the morning urine sample in critically ill children.

A series of pathogenetic events are responsible for transition from sepsis to severe sepsis, septic shock, and organ dysfunction. The infection-induced cellular activation interacts with vascular endothelium leading to release of various cytokines. This results in microvascular injury, thrombosis, and capillary leak, resulting in hypoxic-ischemia injury leading to MODS. ${ }^{31,32}$ Our study demonstrated statistically significant rise of ACR with the increase in the severity of sepsis. This finding is supported by the pathogenetic events of progressive microcirculatory damage with increasing severity of sepsis. Higher levels of ACR were reported in children with sepsis as compared to the patients with noninfectious illnesses. ${ }^{18}$ ACR levels were found be significantly high in children with bacterial meningitis and associated complications. ${ }^{14}$ In a pilot study on adult patients in emergency department with primarily sepsis or severe sepsis, increasing ACR was associated with disposition to higher levels of care. ${ }^{33}$

The MAU has been correlated with disease severity and organ dysfunction scores in adults and children. ${ }^{18,19,34-36}$ In the trauma patients, ACR has been correlated with trauma-related scores. $9,10,37$ ACR was predicted onset of postoperative sepsis and was correlated significantly with sequential organ failure assessment (SOFA) score. ${ }^{38}$ Patients suffering from medical illnesses with increasing ACR had a significantly higher mortality rate and correspondingly higher APACHE II and SOFA scores than those patients with a decreasing ratio. ${ }^{19,34}$

There are very few pediatric studies on the outcome prediction values of ACR. The presence of urinary albumin excretion value $\geq 15$ $\mu \mathrm{g} / \mathrm{minute}$ at 25 to 48 hours had $42 \%$ sensitivity, $85 \%$ specificity, 79\% PPV, and 53\% NPV to indicate a more severe course of bacterial meningitis. ${ }^{14}$ In a study of 41 children, ACR significantly was correlated with PELOD score on day $1(r-0.85 ; p<0.0001)$ and children with MODS had significantly higher ACR ( $p<0.0001)$. An increasing ACR during the first 24 hours of admission had PPV 
Table 4: Correlation of albumin:creatinine ratio with disease severity score, organ dysfunction, and microcirculation parameters

\begin{tabular}{lrrrrrc}
\hline & \multicolumn{3}{c}{ Survivor } & & \multicolumn{2}{c}{ Nonsurvivor } \\
\cline { 2 - 3 } \cline { 6 - 7 } Parameter & \multicolumn{1}{c}{$r s$} & $p$ value & & rs & pvalue \\
\hline PRISM 12-ACR2 & 0.52 & $<0.001$ & & 0.68 & $<0.001$ \\
PRISM 24-ACR & 30.57 & $<0.001$ & & 0.26 & 0.16 \\
PELOD 0-ACR & 10.38 & $<0.001$ & & 0.44 & 0.01 \\
PELOD 12-ACR & 20.48 & $<0.001$ & & 0.73 & 0.001 \\
PELOD 24-ACR & 30.58 & $<0.01$ & & 0.62 & $<0.001$ \\
pH 0-ACR1* & -0.31 & 0.001 & & -0.38 & 0.04 \\
pH 12-ACR2 & -0.14 & 0.14 & & -0.47 & 0.012 \\
pH 24-ACR3 & -0.02 & 0.82 & & -0.56 & 0.002 \\
Lactate 0-ACR1* & 0.04 & 0.65 & & 0.33 & 0.07 \\
Lactate 12-ACR2 & 0.20 & 0.06 & & 0.56 & 0.003 \\
Lactate 24-ACR3 & 0.06 & 0.51 & & 0.41 & 0.03 \\
BD 0-ACR1* & -0.06 & 0.5 & & -0.36 & 0.05 \\
BD 12-ACR2 & 0.04 & 0.65 & & -0.43 & 0.02 \\
BD 24-ACR3 & -0.08 & 0.42 & & -0.20 & 0.29 \\
\hline
\end{tabular}

*Arterial blood sample; PRISM, pediatric risk of mortality score at 12 and 24 hours; PELOD, pediatric logistic organ dysfunction score at the time of admission, 12 hours, and 24 hours of admission; ACR1, ACR2, ACR3 albumin:creatinine ratio ( $\mathrm{mg} / \mathrm{g}$ of creatinine) at the time of admission, 12 hours, and 24 hours of admission, respectively; BD, base deficit

$75 \%$ and NPV $100 \%$ for a higher PELOD score on days 2 or $3 .{ }^{39}$ In a retrospective study of 63 burn ICU pediatric patients, mean ACR value was correlated with length of ICU stay and peak ACR value was also correlated with length of PICU, and hospital stay, severity of burns injury, and organ dysfunction. ${ }^{40} \mathrm{ACR}$ showed a progressive increase during surgery and a decrease afterward, reaching preoperative values within 24 hours after the end of surgery and was also significantly correlated with severity of surgical trauma. ${ }^{41}$ In a study on children with admission diagnoses of sepsis, noninfectious SIRS and diseases without SIRS reported the predictive value of ACR obtained at the time of admission and at 24 hours similar to that of Pediatric Index of Mortality II, PRISM score, PELOD score, and inotrope scores. ${ }^{18}$

We also constructed predicted probability of mortality curves of ACR at the time of admission, 12 hours, and 24 hours of admission to clearly show the increasing mortality with the rising levels of ACR. MacKinnon et al. presented similar curves using multiple regression model and observed significance of urine ACR measured at 6 hours after the admission in adults. ${ }^{36}$ We also identified the level of ACR $>102 \mathrm{mg} / \mathrm{g}$ at the time of admission, 12 hours, and 24 hours of admission significantly associated with morbidity and mortality. At a cutoff value of $101 \mathrm{mg} / \mathrm{g}$, the MAU measured at 24 hours of admission had a sensitivity of $69 \%$, specificity $67 \%$, PPV 31\%, and NPV 91\% for predicting mortality in the critically ill adult patients. ${ }^{17}$ These operative characteristics are similar to our results. In another study, among survivors, the patients with ACR $>100 \mathrm{mg} / \mathrm{g}$ stayed approximately 5 days longer in the hospital and risk of death was 2.7 times as compared with those with $A C R<100$ $\mathrm{mg} / \mathrm{g} .{ }^{35}$ In a pediatric study, an ACR cutoff level of $34.2 \mathrm{mg} / \mathrm{g}$ at the time of admission and $31.6 \mathrm{mg} / \mathrm{g}$ at 24 hours differentiated survivors and nonsurvivors. ${ }^{18}$ These results were not in agreement with the present and previously mentioned adult studies. The probable reason for such low cutoff levels is that 38 (37.2\%) children were without SIRS. The reported median values of ACR at the time of

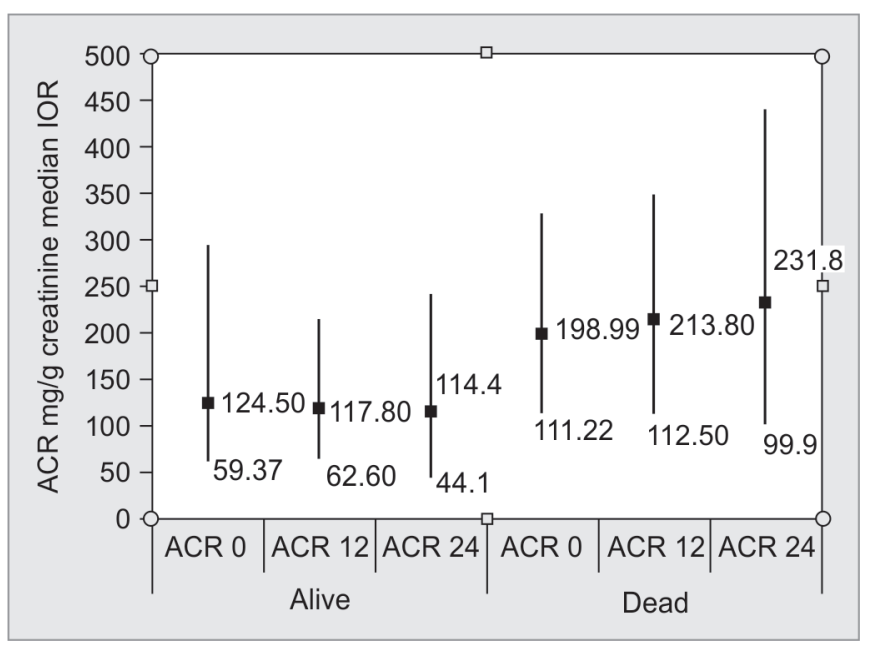

Fig. 1: Comparison of median (IQR) values of albumin:creatinine ratio at the time of admission (ACR1), 12 hours (ACR2), and 24 hours (ACR3) of admission in survivors and nonsurvivors. In survivors, ACR1 124.5(59.4-294.5) vs ACR2 117.8(62.6-211.9), $p$ value 0.008; ACR1 vs ACR3 114.4(44.1-240.3) $p$ value 0.016; ACR2 vs ACR3 $p$ value 0.19. In nonsurvivors, ACR1 198.99(111.22-329.41) vs ACR2 198.99 (111.22329.41), $p$ value 0.19; ACR1 vs ACR3 198.99 (111.22-329.41), $p$ value 0.23 ; ACR2 vs ACR3 $p$ value 0.85

admission and at 24 hours in this particular subset of cases were 6.3 (IQR 3.1-22) mg/g and 4.9 (IQR 2.6-18.7) mg/g, respectively. It is obvious that in the absence of SIRS the endothelial disruption and increased capillary permeability leading to albumin leak are not expected to occur. Authors did not analyze the subgroups of patients with sepsis and with noninfectious SIRS. Other important reasons for variable cutoff level of threshold values include the differences in the discriminative values of the index test, timing of sampling, and means of quantifying the index test. ${ }^{20}$

The present study is the first to investigate the association of $\mathrm{ACR}$ and markers of microcirculation like arterial blood $\mathrm{pH}$, serum lactate, and base deficit. The pathobiology of sepsis discussed previously supports a significant relationship between MAU and these parameters. One may extrapolate that serial estimation of ACR in children with sepsis points towards about the microcirculation status.

The cost analysis and comparison presented in our study clearly indicate the economic advantages. The ACR estimation is 7 times cheaper than one-time estimation of PRISM and PELOD scores. The total expenses will escalate according to the number of times these scores are calculated. The ACR estimation can be done at level I and II critical care units in smaller hospitals as a screening tool at the time of admission and after 24 hours. If the ACR levels were rising along with worsening clinical condition of the patient, it might be used for triage and also for effective and prioritized resource utilization and early transportation to referral hospital.

The strengths of our study are as follows: (a) first study on association between ACR and mortality and morbidity in children admitted in PICU with different severities of sepsis; (b) serial estimation of ACR was performed in all the enrolled patients at a predefined time interval. This provided trends of ACR in survivors and expired patients; and (c) Our study clearly demonstrated a significant relationship between $A C R$ and microcirculation parameters. There are limitations on our study. We did not 
Table 5: Morbidity and mortality analysis with albumin:creatinine ratio (ACR) at the time of admission, 12 hours, and 24 hours of admission with $102 \mathrm{mg} / \mathrm{g}$ cutoff level

\begin{tabular}{|c|c|c|c|c|c|c|c|c|c|}
\hline \multirow[b]{3}{*}{ Parameter } & \multicolumn{2}{|c|}{$A C R 1$} & & \multicolumn{2}{|c|}{$A C R 2$} & & \multicolumn{2}{|c|}{ ACR3 } & \multirow[b]{3}{*}{$p$ value } \\
\hline & $\leq 102$ & $>102$ & \multirow[b]{2}{*}{$p$ value } & $\leq 102$ & $>102$ & \multirow[b]{2}{*}{$p$ value } & $\leq 102$ & $>102$ & \\
\hline & $(n=48)$ & $(n=90)$ & & $(n=50)$ & $(n=88)$ & & $(n=56)$ & $(n=82)$ & \\
\hline Inotrope use ${ }^{\mathrm{a}}(n=54)$ & 9 & 45 & $<0.001$ & 12 & 42 & 0.006 & 15 & 37 & 0.01 \\
\hline \multicolumn{10}{|l|}{ No. of inotropes } \\
\hline$\leq 2$ & 7 & 17 & & 9 & 15 & & 10 & 14 & \\
\hline$>2$ & 2 & 28 & 0.016 & 3 & 27 & 0.015 & 5 & 23 & 0.007 \\
\hline MV Adm $(n=105)$ & 26 & 79 & $<0.001$ & 32 & 73 & 0.01 & 37 & 68 & 0.02 \\
\hline$M V \geq 48 h^{b}(n=93)$ & 20 & 73 & $<0.0001$ & 27 & 66 & 0.01 & 28 & 63 & 0.0002 \\
\hline PICU days ${ }^{c}$ & $5(3-11.7)$ & $6(4-13.2)$ & 0.09 & $6(3-10.2)$ & $6.5(4-14)$ & 0.1 & $5(3-7)$ & $17(4-15)$ & 0.001 \\
\hline Mortality & 4 & 25 & 0.008 & 5 & 24 & 0.01 & 9 & 20 & 0.2 \\
\hline
\end{tabular}

aTwo patients were off inotropes after 24 hours of admission so the number of patients analyzed were 52

${ }^{\mathrm{b}}$ Two patients were off mechanical ventilation within 48 hours in ACR3 group

'Median with interquartile range, ACR1, ACR2, CR3 albumin:creatinine ratio at the time of admission, 12 hours, and 24 hours of admission; MV, mechanical ventilation; Adm, admission; PICU, pediatric intensive care unit
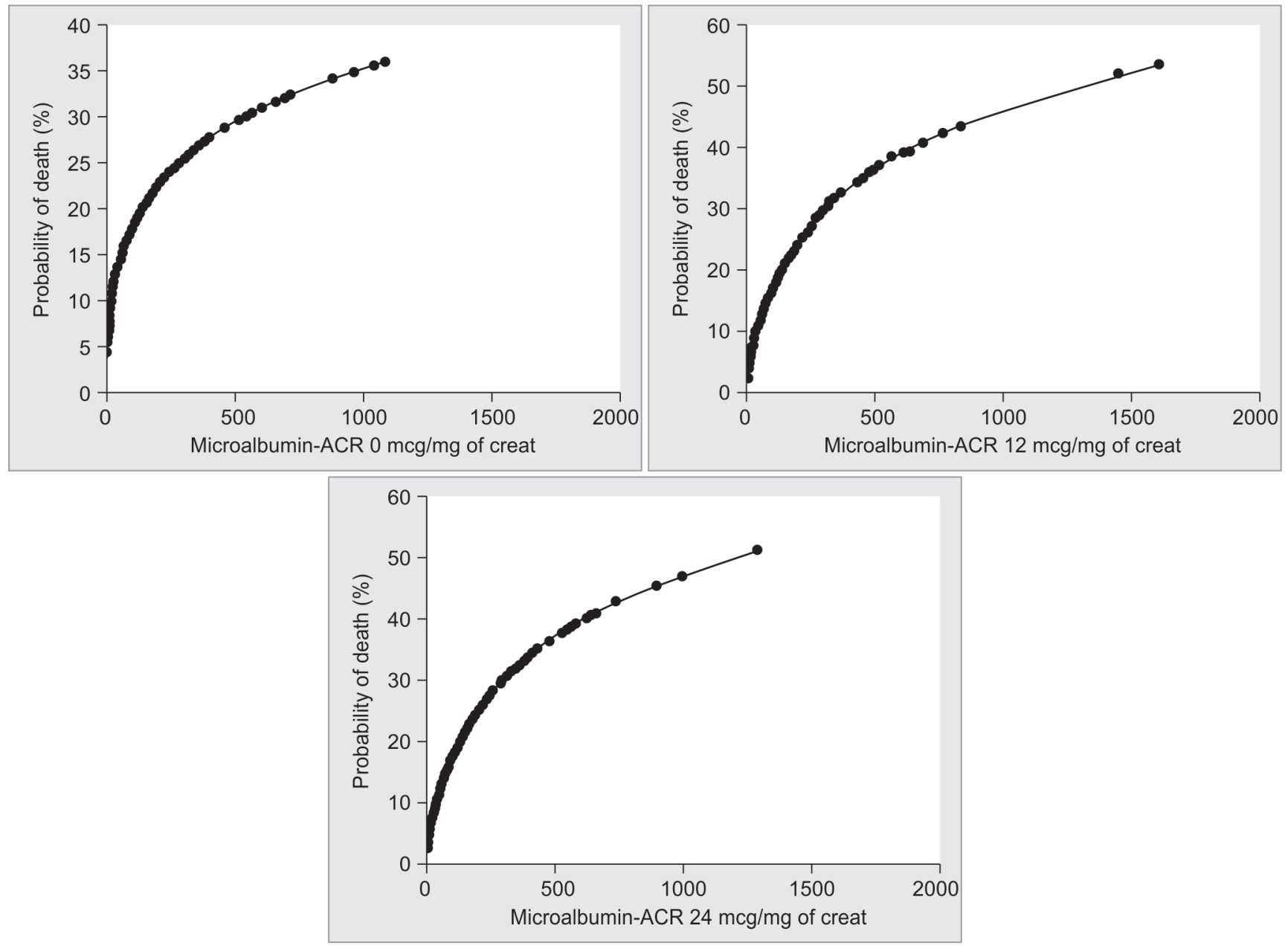

Fig. 2: Predicted probability of death obtained from albumin:creatinine ratio at the time of admission (ACR1), 12 hours (ACR2), and 24 hours (ACR3) of admission using logistic regression

study the effect of muscle mass, cardiac dysfunction, and drugs particularly steroids on $\mathrm{ACR} .{ }^{42-44}$ The results of our study may not be applicable to the patients admitted with clinical conditions without SIRS.
In conclusion, elevated urinary ACR is associated with the severity of sepsis, morbidity, and mortality. The urinary ACR estimation is an easy, cheap, and noninvasive tool and may be used to identify high-risk sepsis cases in resource-settings. Further 
multicenter studies in a different set of population are required to validate our results.

\section{References}

1. Baue $A E$, Durham R, Faist E. Systemic inflammatory response syndrome (SIRS), multiple organ dysfunction syndrome (MODS), multiple organ failure (MOF): Are we winning the battle? Shock 1998;10(2):79-89. DOI: 10.1097/00024382-19980800000001.

2. Davies MG, Hagen PO. Systemic inflammatory response syndrome. Br J Surg 1997;84(7):920-935. DOI: 10.1002/bjs.1800840707.

3. Fleck A, Hawker F, Wallace PI, Raines G, Trotter J, Ledingham IM, et al. Increased vascular permeability: A major cause of hypoalbuminemia in disease and injury. Lancet 1985;325(8432):781-783. DOI: 10.1016/ S0140-6736(85)91447-3.

4. Zikria BA, Bascom JU. Mechanisms of multiple organ failure. In: Reperfusion Injuries and Clinical Capillary Leak Syndrome Zikria BA, Oz MO, Carlson RW Armonk, NY: Futura; 1994. pp 443-492.

5. Feldt-Rasmussen B. Microalbuminuria, endothelial dysfunction and cardiovascular risk. Diabetes Metab 2000;26(S4):64-66.

6. Pedrinelli R, Dell'Omo G, Penno G, Mariani M. Non-diabetic microalbuminuria, endothelial dysfunction and cardiovascular disease. Vasc Med 2001;6(4):257-264. DOI: 10.1177/1358836X0100600410.

7. Smith FCT, Gosling P, Sanghera K, Green MA, Paterson IS, Shearman $\mathrm{CP}$. Microproteinuria predicts the severity of systemic effects of reperfusion injury following infrarenal aortic aneurysm surgery. Ann Vasc Surg 1994;8(1):1-5. DOI: 10.1007/BF02133398.

8. Gosling P, Sanghera S, Dickson G. Generalised permeability defect associated with pulmonary impairment in patients following serious trauma. J Trauma 1994;36(4):477-478. DOI: 10.1097/00005373199404000-00002.

9. Pallister I, Gosling P, Alpar K, Bradley S. Prediction of posttraumatic adult respiratory distress syndrome by albumin excretion rate eight hours after admission. J Trauma 1997;42(6):1056-1061. DOI: 10.1097/00005373-199706000-00012.

10. Raffaele DA, Rosario S, Alessandro DF, Marco F. Glomerular permeability and trauma: A correlation between microalbuminuria and injury severity score. Crit Care Med 1999;27(10):2105-2108. DOI: 10.1097/00003246-199910000-00004.

11. Tisi PV, Shearman CP, Gosling P. Urinary microalbuminuria as a marker for intermittent claudication. Eur J Vasc Surg 1997;13(2):253. DOI: 10.1016/S1078-5884(97)80035-4.

12. Gosling P, Hughes EA, Reynolds TM, Fox JP. Microalbuminuria is an early response following acute myocardial infarction. Eur Heart J 1991;12(4):508-513. DOI: 10.1093/oxfordjournals.eurheartj.a059931.

13. Shearman $\mathrm{CP}$, Gosling $\mathrm{P}$, Walker KJ. Is low proteinuria an early predictor of severity of acute pancreatitis? J Clin Pathol 1989;42(11):1132-1135. DOI: $10.1136 / j c p .42 .11 .1132$.

14. Roinel.Microalbuminuria: An index of severity in childhood meningitis. Pediatr Infect Dis J 1993;12(7):584-588. DOI: 10.1097/00006454199307000-00008.

15. Wood PR, Gosling P, Cook MC. Microalbuminuria following anaphylaxis with general anaesthesia. $\mathrm{Br} J$ Anaesth 2000;84(6): 808-810. DOI: 10.1093/oxfordjournals.bja.a013599.

16. Mahmud N, Stinson J, O'Connell MA, Mantle TJ, Keeling PW, Feely $\mathrm{J}$, et al. Microalbuminuria in inflammatory bowel disease. Gut 1994;35(11):1599-1604. DOI: 10.1136/gut.35.11.1599.

17. Basu S, Chaudhuri S, Bhattacharyya M, Chatterjee TK, Todi S, Majumdar A. Microalbuminuria: An inexpensive, noninvasive bedside tool to predict outcome in critically ill patients. Indian Journal of Clinical Biochemistry 2010;25(2):146-152. DOI: 10.1007/s12291-0100027-9.

18. Anil AB, Anil M, Yildiz M, Kamit Can F, Bal A, Gokalp G, et al. The importance of microalbuminuria in predicting patient outcome in a PICU. Pediatr Crit Care Med 2014;15(5):e220-e225. DOI: 10.1097/ PCC.0000000000000113.
19. Abid O, Sun Q, Sugimoto K, Mercan D, Vincent JL. Predictive value of microalbuminuria in medical ICU patients: Results of a pilot study. Chest 2001;20(6):1984-1988. DOI: 10.1378/chest.120.6.1984.

20. Gopal S, Carr B, Nelson P. Does microalbuminuria predict illness severity in critically ill patients on the intensive care unit? A systematic review. Crit Care Med 2006;34(6):1805-1810. DOI: 10.1097/01. CCM.0000217922.75068.EA.

21. Goldstein B, Giroir B, Adrienne Randolph A. Members of the International Consensus Conference on Pediatric Sepsis International pediatric sepsis consensus conference: Definitions for sepsis and organ dysfunction in pediatrics. Pediatr Crit Care Med 2005;6(1):2-8. DOI: 10.1097/01.PCC.0000149131.72248.E6.

22. Pollack MM, Ruttiman UE, Getson PR. The pediatric risk of mortality (PRISM) score. Crit Care Med 1988;16(11):1110-1116. DOI: 10.1097/00003246-198811000-00006.

23. Leteurtre S, Martinot A, Duhamel A, Proulx F, Grandbastien B, Cotting $J$, et al. Validation of the paediatric logistic organ dysfunction (PELOD) score: prospective, observational, multicentre study. Lancet 2003;362(9379):192-197. DOI: 10.1016/S0140-6736(03)13908-6.

24. Tsioufis C, Mazaraki A, Dimitriadis K, Stefanidis CJ, Stefanadis C. Microalbuminuria in the paediatric age: current knowledge and emerging questions. Acta Paediatr 2011;100(9):1180-1184. DOI: 10.1111/j.1651-2227.2011.02291.x.

25. Gaies MG, Gurney JG, Yen AH, Napoli ML, Gajarski RJ, Ohye RG, et al. Vasoactive-inotropic score as a predictor of morbidity and mortality in infants after cardiopulmonary bypass. Pediatr Crit Care Med 2010;11(2):234-238. DOI: 10.1097/PCC.0b013e3181b806fc.

26. Gosling P. Microalbuminuria: a sensitive indicator of nonrenal disease? Ann Clin Biochem 1995;32(5):439-441. DOI: 10.1177/000456329503200501.

27. Evans G, Greaves I. Microalbuminuria as a predictor of outcome: Shows promise but large prospective trials are needed. BMJ 1999;318(7178):207-208. DOI: 10.1136/bmj.318.7178.207.

28. Gosling P, Manji M, Czyz J. Microalbuminuria: timing is everything!. Inten Care Med 2003;29(8):1394. DOI: 10.1007/s00134-003-1817-9.

29. Bakker AJ. Detection of microalbuminuria: Receiver operating characteristic curve analysis favours albumin-to-creatinine ratio over albumin concentration. Diabetes Care 1999;22(2):307-313. DOI: 10.2337/diacare.22.2.307.

30. Jones CA, Francis ME, Eberhardt MS, Chavers B, Coresh J, Engelgau $M$, et al. Microalbuminuria in the US population: Third National Health and Nutrition Examination Survey. Am J Kidney Dis 2002;39(3): 445-459. DOI: 10.1053/ajkd.2002.31388.

31. Nguyen HB, Rivers EP, Abrahamian FM, Moran GJ, Abraham E, Trzeciak $S$, et al. Severe sepsis and septic shock: Review of literature and emergency department management guidelines. Ann Emerg Med 2006;48(1):28-54. DOI: 10.1016/j.annemergmed.2006.02.015.

32. Angus DC, van der Poll T. Severe sepsis and septic shock. N Engl J Med 2013;369(9):840-851. DOI: 10.1056/NEJMra1208623.

33. Drumheller BC, McGrath M, Matsuura AC, Gaieski DF. Point-ofcare urine albumin:creatinine ratio is associated with outcome in emergency department patients with sepsis: A pilot study. Acad Emerg Med 2012;19(3):259-264. DOI: 10.1111/j.1553-2712.2011.01266.x.

34. Thorevska N, Sabahi R, Upadya A, Manthous C, AmoatengAdjepong Y. Microalbuminuria in critically ill medical patients: Prevalence, predictors, and prognostic significance. Crit Care Med 2003;31(4):1075-1081. DOI: 10.1097/01.CCM.0000059316.90804.0B.

35. Gosling P, Brudney S, McGrath L, Riseboro S, Manji M. Mortality prediction at admission to intensive care: A comparison of microalbuminuria with acute physiology scores after 24 hours. Crit Care Med 2003;31(1):98-103. DOI: 10.1097/00003246-20030100000016.

36. MacKinnon KL, Molnar Z, Lowe D, Shearer E. Use of microalbuminuria as a predictor of outcome in critically ill patients. $\mathrm{Br} J$ Anaesth 2000;84(2):239-241. DOI: 10.1093/oxfordjournals.bja.a013409.

37. Pallister I, Dent C, Wise CC, Alpar EK, Gosling P. Early post-traumatic acute respiratory distress syndrome and albumin excretion rate: 
A prospective evaluation of a "point-of-care" predictive test. Injury 2001;32(3):177-181. DOI: 10.1016/s0020-1383(00)00149-2.

38. De Gaudia AR, Adembri C, Grechi S, Novelli GP. Microalbuminuria as an early index of impairment of glomerular permeability in postoperative septic patients. Intensive Care Med 2000;26(9): 1364-1368. DOI: 10.1007/s001340000593.

39. Wakeham MK, Rajzer KL, Angst DB, Torero LE, Jamovich DG. Microalbuminuria levels are correlated with PELOD scores in critically ill children. Pediatr Crit Care Med 2004;5(5):509. DOI: 10.1097/00130478-200409000-00045.

40. Din AH, Frew Q, Smailes ST, Dziewulski P. The utility of microalbuminuria measurements in pediatric burn injuries in critical care. J Crit Care 2015;30(1):156-161. DOI: http://dx.doi.org/10.1016/j.jcrc.2014.09.005.

41. Sarti A, De Gaudio AR, Messineo A, Cuttini M, Ventura A. Glomerular permeability after surgical trauma in children: Relationship between microalbuminuria and surgical stress score. Crit Care Med 2001;29(8):1626-1629. DOI: 10.1097/00003246-20010800000021.

42. Cirillo M, Laurenzi M, Mancini M, Zanchetti A, De Santo NG. Low muscular mass and overestimation of microalbuminuria by urinary albumin/creatinine ratio. Hypertension 2006;47(1):56-61. DOI: 10.1161/01.HYP.0000197953.91461.95.

43. Katz DH, Burns JA, Aguilar FG, Beussink L, Shah SJ. Albuminuria is independently associated with cardiac remodelling, abnormal right and left ventricular function, and worse outcomes in heart failure with preserved ejection fraction. J A C C: Heart Failure 2014;2:586-596.

44. Rinaldi S, Adembri C, Lla Grechi S, De Gaudio AR. Low-dose hydrocortisone during severe sepsis: Effects on microalbuminuria. Crit Care Med 2006;34(9):2334-2339. DOI: 10.1097/01.CCM 0000233872.04706.BB. 